\title{
Keine Inspektionen, sondern ein sinnvolles Miteinander
}

\author{
V. Schreiber
}

Die neue Weiterbildungsordnung stösst nicht überall auf Begeisterung. Aufwand und Kosten für Weiterbildungskonzepte und Visitationen seien zu hoch, wird kritisiert. Beide Instrumente können aber - sinnvoll eingesetzt - für alle Beteiligten von Vorteil sein.

\footnotetext{
* In diesem Artikel werden männliche Formen verwendet. Selbstverständlich sind damit sowohl weibliche wie auch männliche Formen gemeint.
}

Korrespondenz: Vital Schreiber Mitglied des Geschäftsausschusses VSAO

Ko-Leiter Ressort Weiterbildung Glärnischstrasse 10 CH-8700 Küsnacht
Während seiner beruflichen Entwicklung durchschreitet ein Arzt* drei Stufen: die Ausbildung zum Arzt (Studium), die Weiterbildung zum Facharzt (Anstellung meist als AA oder junger OA) und die Fortbildung, neu zur Aufrechterhaltung des Facharzttitels FMH (Veranstaltungen, Seminare, Kongresse). Um ein möglichst guter Arzt zu werden oder zu sein, müssen alle drei Stufen einen entsprechenden Qualitätsstandard aufweisen. Der VSAO kümmert sich insbesondere um eine qualitativ hochstehende und inhaltlich wie zeitlich machbare Weiterbildung in einem sinnvollen Arbeitsumfeld. Eines der Mittel, die Weiterbildungsqualität zu erhalten oder gar zu verbessern, sind die Weiterbildungskonzepte und die Visitationen.

\section{Weiterbildungskonzepte}

Die neue Weiterbildungsordnung (WBO), die durch die Ärztekammer gutgeheissen wurde, schreibt den Weiterbildungsstätten (WB-Stätten) vor, ein Weiterbildungskonzept (WB-Konzept) zu erarbeiten und $\mathrm{zu}$ befolgen. Die FMH hat den Fachgesellschaften ein empfohlenes Raster gesandt. Diese wiederum haben es ihrer Disziplin angepasst und allen WB-Stätten (Spitälern, Kliniken und Instituten) weitergesandt. Jene hätten bis zum 31. Dezember 2002 ein WB-Konzept erarbeiten und veröffentlichen sollen. Diese Konzepte bilden die Grundlage für die Visitationen.

\section{Visitationen}

Unter Visitation versteht man den Besuch einer WB-Stätte durch einen Vertreter der FMH, einen Vertreter der entsprechenden Fachgesellschaft und einen Vertreter des VSAO. Die Visitation sollte alle 7 Jahre oder nach dem Wechsel des Leiters der WB-Stätte oder nach Anfrage einer der drei erwähnten Gesellschaften erfolgen. Insge- samt rechnen wir somit gesamtschweizerisch mit ungefähr 200 Visitationen pro Jahr. Die Visitation gilt lediglich der Überprüfung des WB-Konzeptes, welches vorgängig durch die WB-Stätte veröffentlicht wurde. Sie beinhaltet einen Besuch der Institution mit Einblick in Rapporte, Visitenstrukturen, Arbeitsabläufe. Hinzu kommen je ein Gespräch mit dem Chefarzt, den Leitenden Ärzten, Oberärzten und den Assistenzärzten sowie die Einsichtnahme in die überprüfbaren, objektiven WB-Werte oder Leistungen, wie zum Beispiel die einzelnen Logbücher der Ärzte in WB. Die Visitation sollte mit einem Gespräch im Plenum enden. Anschliessend werden die Erkenntnisse in einem Schlussbericht zusammengefasst. Neu kann sich nun ein erkannter und in der Folge nicht behobener ausgeprägter Missstand auf die Einstufung der WB-Stätte auswirken. Diese Regel gilt für alle Institutionen. Wesentlich für das Gelingen der Visitationen ist die Erkenntnis, dass es bei einer Visitation um einen interaktiven und konstruktiven Ideenaustausch geht. Versucht werden sollte, mögliche Verbesserungsvarianten aufzuzeigen und im Verlauf umzusetzen. Die Fachgesellschaft für Anästhesie war mit diesem System, schon bevor es zur Pflicht wurde, vertraut und hat im konstruktiven Austausch gute Ergebnisse erzielt.

\section{Vor- und Nachteile des Systems}

Vorteil der WB-Konzepte ist, dass die Fachgesellschaften fachspezifische Anliegen integrieren können. Entsprechend kann auch jede Institution aktiv und selbständig ein WB-Konzept entwickeln, welches auf ihre lokalen Verhältnisse zugeschnitten ist. Besteht der Wille zur Weiterbildung von jungen Ärzten, tragen die im WBKonzept festgelegten Überlegungen zur Verbesserung der Weiterbildung bei. Zudem werden die Organisationsabläufe klargestellt und vereinfacht.

Der systembedingte Nachteil der Visitation ist, dass diese lediglich die Einhaltung des WB-Konzeptes überprüft und nicht dessen Inhalt. Es wird sich im Verlauf zeigen, ob es den Besuchern aus den drei Interessensgruppen gelingen wird, durch eine zweckmässige Gestaltung 
der Visitation auch Einfluss auf das WB-Konzept zu nehmen. So ist es dem VSAO ein besonderes Anliegen, dass in den WB-Konzepten klar definiert wird, welche Aufgaben, Funktion und Aussichten ein Arzt in Weiterbildung hat. Spannungen im Ärzteteam können auf diese Weise reduziert werden. Die Motivation kann gesteigert, die Unzufriedenheit oder Ernüchterung reduziert werden. Ein klares WB-Konzept für den Stelleninhaber - bereits vor Stellenantritt - hilft auch den Institutionen. Dies betrifft insbesondere jene Institutionen mit einem grösseren Dienstleistungs- oder gar Forschungsauftrag, die dafür mehr Ärzte benötigen als direkt weitergebildet werden können. Der VSAO sieht gerade dort die Möglichkeit, Spitalärzte einzusetzen. Auch wenn diese - zugegebenermassen - noch nicht in grosser Zahl auf dem Arbeitsmarkt zu finden sind. Darüber hinaus bietet sich die Gelegenheit, die dringend notwendige Rationalisierung der Arbeitsinhalte und -abläufe vorzunehmen. Eine Massnahme, welche den Ärztebedarf deutlich senken würde und wahrscheinlich - als sekundärer Gewinn - zur ebenso dringend notwendigen Steigerung des Marktwertes des Arztes führen würde. Die Visitationen ermöglichen uns, solche Überlegungen direkt dem Leiter einer WB-Stätte zu übermitteln und ihn im persönlichen Kontakt auch zu motivieren. Zurzeit haben wir nämlich keine andern Mittel, diese Forderungen voranzutreiben. Die Gefahr besteht, dass einzelne Leiter von WB-Stätten die in den Konzepten festgelegten Anforderungen zum Vorwand nehmen, die WB-Zeit zu verlängern. Dies liegt nicht im Interesse des VSAO.

Um bald eine hohe und überprüfbare Weiterbildungsqualität $\mathrm{zu}$ erreichen, strebt der VSAO auch den Wechsel von infrastrukturellen zu leistungsabhängigen Kriterien für die Einstufung der WB-Stätten an. Neu sollten nicht mehr die Anzahl Kaderärzte, die Anstellungsprozente, die Anzahl Betten und weiteres ausschlaggebend sein, sondern beispielsweise die Anzahl Weiterbildner, die Anzahl instruierter Interventionen oder die Anzahl Weiterbildungen. Derartige Einstufungskriterien finden dann automatisch ihren Niederschlag in den WB-Konzepten, welche anschliessend durch die Visitationen überprüft werden können und sich entsprechend auf die Einstufung der Institution auswirken.

\section{Aktueller Stand}

Bis Ende 2002 haben die meisten - aber noch nicht alle-WB-Stätten WB-Konzepte aufgestellt. Zurzeit werden viele Konzepte noch durch die einzelnen Fachgesellschaften (FG) überprüft. Der VSAO bemüht sich, bereits beim Aufbau der WB-Konzepte bei den FG Einfluss zu nehmen. Ziel ist, bei den Visitationen bereits WBKonzepte anzutreffen, die unseren Schwerpunkten entgegenkommen. Wir fordern die sachliche und zeitliche Machbarkeit des Konzeptes, eine Zielvereinbarung vor, bei und nach Stellenantritt, die regelmässige Betreuung durch facherfahrenere Ärzte, externe und interne Weiterbildungsangebote sowie die klare Aufteilung und Auflistung der Ärzte mit und ohne WB-Stelle. Sicher sind wir aber in den FG beziehungsweise in deren Weiterbildungskommissionen noch zu wenig vertreten.

Nicht überall werden die neue Strukturierung und das kontrollierbare Bekenntnis zur WB begrüsst. Im März 2003 haben sich erschreckend viele FG bei der Kommission für Weiter- und Fortbildung (KWFB) der FMH über das System der WB-Konzepte und der Visitationen beschwert: Viele WB-Stätten scheuen den Mehraufwand; einzelne überlegen sich bereits ernsthaft, keine Weiterbildung mehr anzubieten beziehungsweise den Betrieb nur noch mit Fachärzten zu betreiben. Dies mag die Institutionen kurzfristig entlasten, längerfristig könnte sich die Massnahme kontraproduktiv auswirken. Wir bedauern diese Tendenz, müsste doch die Nachwuchsförderung und das Weitergeben der Erfahrung an junge Kollegen ein wichtiges Anliegen der Ärzteschaft sein.

Streitpunkt sind einerseits die WB-Konzepte: Es sei ein $\mathrm{zu}$ grosser Papierkrieg für wenig Erreichbares, wird moniert. Ein sinnvolles und einhaltbares WB-Konzept zu erstellen, ist zweifellos zeitintensiv. Der grosse Zeitaufwand ist aber einmalig, und nachfolgende Korrekturen oder Anpassung an neue Verhältnisse bedürfen keines grossen Aufwands. Ein klares Konzept benötigt auch keine grossen Ausführungen. Die Behauptung gewisser Fachgesellschaftsvertreter, ohne Konzept seien die Kandidaten stärker motiviert, weisen wir dezidiert zurück. Gerade die schlechte WB-Qualität hat den VSAO dazu bewogen, das System der Konzepte und Visitationen der FMH zu unterstützen und zu fördern.

Kritisiert werden andererseits die Visitationen: Sie seien zu personal- und kostenintensiv. Die Visitationen sind wahrlich personalintensiv. Am stärksten davon betroffen sind aber die KWFB und der VSAO, welche bei jeder Visitation anwesend sein müssen. Für die Fachvertreter hängt der Aufwand von der Anzahl WB-Stätten in der Schweiz ab. Darüber hinaus wird diese Zahl noch durch sieben geteilt, da eine Visitation 
nur alle sieben Jahre vorgesehen ist. Es drängt sich die Vermutung auf, die opponierenden FG hätten sich einfach zu spät mit dieser Aufgabe auseinandergesetzt und gerieten nun in einen Vertreterengpass. Setzt sich jedoch die Einsicht durch, dass die Visitation keine Inspektion der WB-Stätte sein sollte, sondern dem gemeinsamen Streben dient, die WB-Qualität hochzuhalten, wird klar, dass nicht immer der leitende Experte der FG anwesend sein muss, damit der visitierte Leiter einer WB-Stätte die Vorschläge des Visitationsteams annehmen kann.

Schliesslich stellt sich die Frage nach den Kosten. Diese ist berechtigt, denn nach dem ersten Jahr sollten die WB-Stätten die Kosten der Visitationen übernehmen. Der finanzielle Aspekt wirft grundsätzliche Fragen auf: Wie viel ist uns die Weiterbildung zeitlich, personell und finanziell wert? Sie betrifft sowohl uns ausgebildete und sich in Weiterbildung befindende Ärzte als auch uns Patienten und Bürger. Wer hat welche Kosten in welcher Form zu tragen? Ist es der Betrieb und der Staat oder die Ärzteschaft und die Weiterzubildenden oder die Krankenkassen und die Patienten? Ist in einem Betrieb die Weiterbildung von der Ausbildung, der Fortbildung und der Berufserfahrung exakt unterscheidbar? Muss für die Weiterbildung Geld oder Arbeitszeit eingesetzt werden? Zumindest für die Visitationen fallen die Kosten für die WB-Stätten mit der Entschädigung von drei Vertretern alle sieben Jahre wahrlich nicht so hoch aus.

\section{Aufwand und Ertrag}

Schon früh haben die zwei Ressortleiter WB des Geschäftsausschusses VSAO, Susi Stöhr und Vital Schreiber, erkannt, dass eine grosse Zahl genügend berufserfahrener und fachkompetenter junger Ärzte nötig sein wird, um an allen Visitationen teilnehmen zu können. Daher suchen wir bereits seit Ende 2001 VSAO-Mitglieder, die gewillt sind, einen Nachmittag pro Jahr für eine Visitation aufzuwenden. Mit der FMH konnten wir vereinbaren, dass wir spätestens drei Monate im voraus über eine bevorstehende Visitation orientiert werden. Dies gibt unseren Vertretern genügend Zeit, sich zu organisieren. Um unserem Streben nach einer hohen WB-Qualität gerecht $\mathrm{zu}$ werden, haben wir eine Einführung beziehungsweise Schulung der Visitatoren vorgesehen. Die Visitation wird übrigens vergütet. Der Freistellung durch den eigenen Leiter der WB-Stätte sollte im Rahmen dieser FMH- und Fachgesellschaftsaufgabe nichts im Wege stehen.

Der VSAO befürwortet derzeit uneingeschränkt das System der WB-Konzepte und Visitationen. Am wichtigsten ist für uns die Gewährung, Einhaltung und Überprüfung einer qualitativ hohen und machbaren Weiterbildung. Welches System zum angestrebten Ziel führt, ist uns letztendlich egal. Es lohnt sich aber, die bereits geleisteten, umfangreichen Arbeiten nun umzusetzen und die Visitationen zu starten. Eine Evaluation der Zielerreichung nach einer gewissen Zeit kann dann zu Anpassungen oder $\mathrm{zu}$ einem Systemwechsel führen. Mittelfristig plant der VSAO deshalb eine Überprüfung der Zweckmässigkeit beziehungsweise der Zielerreichung durch die Visitationen.

Ich bin der Überzeugung, dass die Visitationen an sich für die Institutionen und die Besucher bereichernd sein können. Sofern beide Seiten versuchen, sich sachlich der Weiterbildung zu widmen. Der konstruktive Ansatz und die Akzeptanz verschiedener Meinungen und Erfahrungen können zu Verbesserungen der Weiterbildung und der Betriebsorganisation führen, was ein angestrebtes Ziel des VSAO ist. 